\title{
Interactions of Solid Supports and Fluorescent Substances in Thermally Activated Delayed Fluorescence
}

\author{
Yoshiaki OnOuE*, Keizō Hiraki** and Yasuharu NishiKaWA** \\ *Nara-Ikuei Gakuen, Horen, Nara 630 \\ ** Department of Chemistry, Faculty of Science and Technology, Kinki University \\ Kowakae, Higashiosaka 577
}

\begin{abstract}
The effect of the solid support on thermally activated delayed fluorescece (TADF) was investigated. Comparison of the molecular structures of supports and consideration of the energy levels of the absorption band and the fluorescence, and of lifetimes of emissions allowed the postulation of certain molecular criteria for the supports of TADF. The interactions of Tin-protoporphyrin IX dimethyl ester (Sn-PPDE) with a polyvinyl alcohol (PVA) were considered in detail. The hydrogen bonds ( $-\mathrm{OH}-\pi$-electron systems of $\mathrm{Sn}$-PPDE) were found to contribute to fix Sn-PPDE rigidly in the PVA matrix, thereby minimizing non-radiative decay of the excited molecule in the triplet state.
\end{abstract}

Keywords Thermally activated delayed fluorescence, tin-protoporphyrin IX dimethyl ester, polyvinyl alcohol

Thermally activated delayed fluorescence (TADF) has a relatively long radiative lifetime, almost equal to that of phosphorescence. Therefore, loss of excitation energy by collisional transfer is greatly enhanced. But it is possible to minimize collisional quenching by fixing molecules on stationary phases. ${ }^{1}$ In fact, although TADF of porphyrins is rarely observed at room temperature in solution, substances adsorbed on filter paper or placed in boric acid glasses become good luminous molecules of TADF. ${ }^{2}$ Thus the interaction between solid support and fluorescent substance is an important factor in order to restrict vibrational motions causing the non-radiative decay from the triplet state. But the exact mechanism of interaction between solid support and fluorescent substance is still not completely elucidated. Filter paper is commonly used as the support of TADF because of its easy handling. In this study, polymers, such as polyethylene (PE), polystyrene (PS), polyvinyl chloride (PVC), polyvinyl alcohol (PVA), acetyl cellulose (AC), polyacrylic acid (PA), and polyacryl amide (PAA) were used as the support. Common molecular skeletons of all these polymers are $\mathrm{PE}$, except $\mathrm{AC}$, though each functional group of these polymers is different. Then the effect of the functional group of the solid support on the interaction between the support and fluorescent substance of TADF was investigated.

\section{Experimental}

\section{Reagents}

PVA 2000 (saponification degree 78-82 mol\%), sodium acrylate polymer, $\mathrm{f}_{\mathrm{CH}_{2}} \mathrm{CHCOONa}{ }_{n}$, $n=22000-66000$, and vinyl chloride polymer ${ }_{\mathrm{f}} \mathrm{CH}_{2} \mathrm{CHCl}{ }_{n}, n=1020$ were purchased from Kishida Chemical Co. AC (M. Woelm, Eschwege, Germany) and PE (Art. 7422 MERCK) were used. Tinprotoporphyrin IX dimethyl ester complex (Sn-PPDE) was synthesized as described in an earlier publication. ${ }^{3}$ All other chemicals used in this study were of analytical reagent grade and were used without further purification.

\section{Preparation of PVA film containing Sn-PPDE}

Stock solution of $\mathrm{Sn}-\mathrm{PPDE}\left(1 \times 10^{-4} \mathrm{~mol} / 1\right.$ in acetone) was prepared. After Sn-PPDE solution (2 $\mathrm{ml}$ ) was added to $10 \mathrm{~g}$ PVA granules, the granules were well ground to powder. The powder was dissolved in $100 \mathrm{ml}$ of water by heating at about $60^{\circ} \mathrm{C}$. After the elimination of foam by ultrasonication, the viscous solution was spread over a glass plate in a uniform layer, and the plate was allowed to stand until the film was solidified. After standing, the film was dried at $60^{\circ} \mathrm{C}$ for $3 \mathrm{~h}$, and then kept in a desiccator.

\section{Preparation of PVC, PS, AC, PA and PAA films}

Granules of PVC, PS and AC were dissolved in cyclohexane, benzene and acetone, respectively, and PA and PAA were dissolved in water. These films containing Sn-PPDE were prepared in the same way as PVA film.

\section{Preparation of PE film}

Granules of PE were thoroughly mixed with the SnPPDE solution, and placed between two glass plates 
and heated. After melting, pressure was exerted on the glass plates to spread PE uniformly (about $0.5 \mathrm{~mm}$ thick).

The films of Sn-PPDE were cut into $1 \times 2 \mathrm{~cm}$ pieces. These pieces were fitted to the holder of TADF measurement. ${ }^{3}$ For TADF, room temperature phosphorescence (RTP) and fluorescence measurements, a Hitachi 650-10S spectrofluorometer equipped with a phosphoroscope attachment was used.

\section{Results and Discussion}

Fluorescences of Sn-PPDE in all films employed in this study were found under irradiation with blue light $(420 \mathrm{~nm})$, but TADF and RTP were observed only in PVA, PA and PAA films. As shown in Fig. 1, the basic structural unit of all resins employed in this study consists of ethylene, except for AC. All resins differ in functional group: i.e. chloride (PVC), benzene (PS), hydroxy (PVA), carboxy (PA) and amide (PAA). Therefore, we can conclude that functional groups have a significant influence on long-lived emission. Thus, the influence of these functional groups was examined by comparison with the energy levels of the absorption band, fluorescence, TADF and RTP of Sn-PPDE enclosed in the films or adsorbed on thin layers of alumina, silica and cellulose. The energy levels of the emissions and the absorption bands were

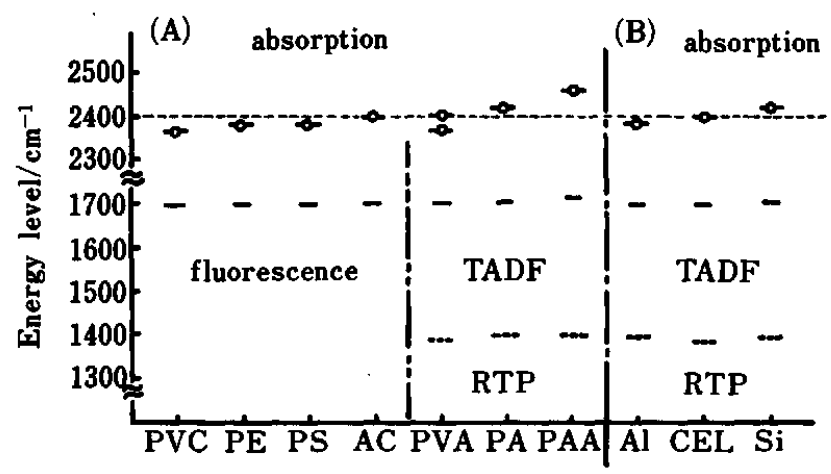

Fig. I Comparisons of energy level of absorption band, fluorescence, thermally activated delayed fluorescence (TADF), and room temperature phosphorescence (RTP) of Sn-protoporphyrin IX dimethyl ester enclosed in seven kinds of films (A), and adsorbed on three kinds of thin layers (B).

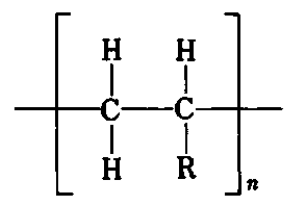

$$
\begin{aligned}
& \text { PE: } R=-H \quad \text { PVA: } R=-O H \\
& \text { PA: } R=-C O O H \\
& \text { PVC: } R=-C \text { PAA: } R=-\mathrm{CONH}_{2} \\
& \text { PS: } R=-\square
\end{aligned}
$$

[Film] PVA: polyvinyl chloride, PE: polyethylene, PS: polystylene, AC: acetyl cellulose, PVA: polyvinyl alcohol, PA: polyacrylic acid and PAA: polyacryl amide.

[Thin layer] Al: alumina, CEL: cellulose and Si: silica gel. evaluated from the wavelength of the maximum of the spectrum. As shown in Fig. 1(A), the energy levels of the absorption band corresponding to the Soret band of Sn-PPDE in PVC, PE, PS and AC are not higher than $2400 \mathrm{~cm}^{-1}$, and neither TADF nor RTP of SnPPDE were observed in these resins. Contrary to these resins, the energy levels of Soret bands of Sn-PPDE in PVA, PA and PAA are higher than $2400 \mathrm{~cm}^{-1}$, in such cases both TADF and RTP were confirmed. The Soret band of porphyrin is due to the electron transfer between $\pi$ and $\pi^{*}$. Therefore, the differences in energy of the absorption maximum of Sn-PPDE in several substances become a good measurement of the difference in energy of the interaction between $\pi$ electron of Sn-PPDE and substance in the ground state. The first requisite of support for the TADF measurement is that the matrix of support holds the adsorbed compound rigid and thereby restricts vibrational energy necessary for non-radiative decay from the triplet state. From this viewpoint, it might be concluded that the relationship between Sn-PPDE and matrices of PVA, PA and PAA make the fluorescent substance well fixed to inhibit non-radiative transition of excimer. Then the relationship between the energy levels of absorption band and the TADF intensity was investigated. The intensity increased in the order of PAA $<$ PA $<$ PVA and the order of increasing of TADF intensity of Sn-PPDE adsorbed on thin layer was silica gel<alumina<cellulose. Contrary to the order of TADF intensity, the order of energy increasing is PVA $<$ PA $<$ PAA for film and alumina $<$ cellulose $<$ silica gel for thin layer. These results indicate that at least the intensity of TADF does not directly relate to the energy level of the absorption band. It was found that the hydroxyl group, in the case of both the film and the thin layer, was the most effective function group of all functional groups employed in this study.

\section{Interaction between hydroxyl group and Sn-PPDE}

Hydroxyl groups of PVA are favorably located so that the protons form hydrogen bands to adjacent $\pi$ electrons of the porphyrin ring. The hydroxyl group is expected to play an important role in fixing the substance firmly enough to inhibit collisional quenching. This suggestion was supported by the following experimental results. As shown in Fig. 1(A), AC film of Sn-PPDE showed the maximum absorption band at $2400 \mathrm{~cm}^{-1}$, but TADF of Sn-PPDE in AC was not observed. This non-luminescence is thought to be responsible for an acetylation of hydroxyl groups. Then the interaction between Sn-PPDE and hydroxyl groups was investigated in detail. As shown in Fig. 1(A), there are two absorption energy levels of SnPPDE only in PVA: the lower one $\left(2370 \mathrm{~cm}^{-1}\right)$ was observed immediately after the sample was irradiated with blue light $(420 \mathrm{~nm})$, then the energy level of the absorption band rises slowly with the illumination time up to the maximum $\left(2405 \mathrm{~cm}^{-1}\right)$. According to the blue shift of the absorption band, the strength of TADF of 


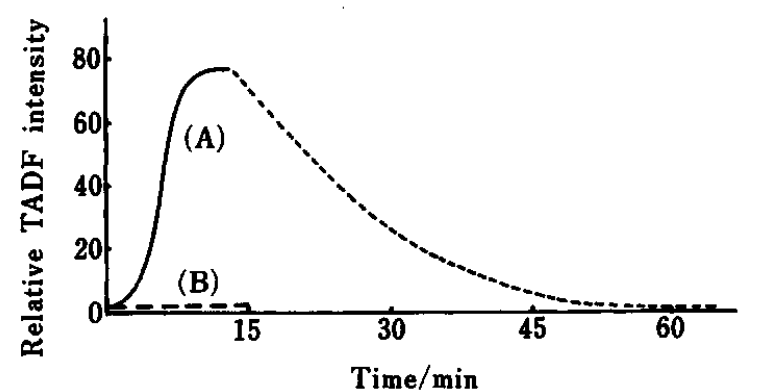

Fig. 2 Dependence of TADF intensity of Sn-PPDE in PVA film on irradiation time. (A): PVA film of Sn-PPDE was successively irradiated with light of $420 \mathrm{~nm}$, until the TADF intensity reached maximum level. After the irradiation for $14 \mathrm{~min}$, TADF intensity was measured at intervals separated by irradiations with light. (B): PVA film of Sn-PPDE was not successively irradiated with light, and TADF intensity was measured at regular time intervals by irradiation with light.

Sn-PPDE drastically increased, as shown in Fig. 2. The strength of TADF goes up to more than 50-fold the initial value within $14 \mathrm{~min}$ at $43.5 \pm 0.5^{\circ} \mathrm{C}$. After attaining the maximum level, this level remains constant so long as the film is irradiated, and the ratio of the intensities of TADF and RTP decreased exponentially with the reciprocal of the absolute temperature following the Arrenius' theory. But once irradiation is stopped, as shown in Fig. 2, the intensity of TADF decreases with the elapse of time, and the intensity of TADF falls to the initial TADF intensity with $45 \mathrm{~min}$. On the same sample, this TADF increase and decrease was repeated by successive irradiation and shielding from the irradiation. Of course, as shown in Fig. 2(B), no TADF increase was observed by an intermittent irradiation with light. This increase of TADF was due to the formation of hydrogen bonds between hydroxyl groups of PVA and $\pi$-electron systems of Sn-PPDE because of the reasonable rising of the energy level of absorption band of Sn-PPDE by irradiation. If it were not for the formation of hydrogen bonds, it is hard to see why such an increase of the energy level of absorption band and such an increase of TADF intensity could be induced. This supposition was supported by the measurements of TADF lifetime. The lifetime which was measured immediately after irradiation with light was $8 \pm 0.5 \mathrm{~ms}$ at $43^{\circ} \mathrm{C}$, and after $15 \mathrm{~min}$ of continuous irradiation, it was prolonged to $18 \pm 0.5 \mathrm{~ms}$ at $43^{\circ} \mathrm{C}$. This prolongation of TADF lifetime was interpreted to be dependent on the increase of the stability of Sn-PPDE in PVA matrix according to the formation of hydrogen bonds, hence the rate constant of radiationless energy loss from the lowest triplet to the ground state was reduced.

As described previously, the increase and decrease of TADF intensity of Sn-PPDE in PVA could be repeatedly observed on the same sample. This indicates that the formation of the hydrogen bonds is reversible. But this increase and decrease of TADF intensity was not observed in the case of cellulose employed as a support of Sn-PPDE, nevertheless cellulose has hydroxyl groups in its molecule in the ratio of one hydroxyl group to two carbons, the same ratio as that of PVA. So the effects of molecular and crystal structure of support on TADF intensity were investigated. Crystallinity of PVA is dependent to a great extent on the degree of saponification, that is, the lower one has some residual acetyl groups in the molecule of PVA, and residual acetyl groups result in a lowering of the crystallinity of PVA due to a steric hindrance of hydrogen bonds between external or internal hydroxyl groups. ${ }^{4}$ Then the effect of saponification degree of PVA on TADF was investigated. The highly saponified PVA $(98.5-99 \mathrm{~mol} \%)$ was compared with the lower one $(78-82 \mathrm{~mol} \%)$ as a support of TADF measurement. The Sn-PPDE film of PVA, which is a higher saponification, was prepared by the same manner as the lower one. The film of high degree does not undergo the drastic increase of TADF by irradiation as observed in the film of lower degree, and the intensity of high degree one was lower than one-third of that of the lower degree. This result implies that the lower crystallinity is favorable for TADF support because the lowly saponified PVA has a large number of free hydroxyl groups which do not form hydrogen bonds. From this viewpoint, the behavior of cellulose as a support of TADF might be understood. Cellulose is insoluble in water, and this insoluble characteristic is mainly responsible for the formation of hydrogen bonds between external or internal molecules, whose hydrogen bonds enhance the crystallinity of the molecule. That is, high crystallinity molecules are fairly limited to having free hydroxyl groups which act as a hydrophylic group. Therefore, no appreciable stabilization of Sn-PPDE in cellulose matrix by the hydrogen bonds between hydroxyl groups of cellulose and $\pi$-electron systems of $\mathrm{Sn}$ PPDE is expected. This supposition was supported by the following three experiments. (1) The behavior of boron-PVA compound as a support for TADF measurement was investigated. The Sn-PPDE film of boron-PVA compound was prepared by the following method. Sodium tetraborate aqueous solution (3\%) was added to the aqueous PVA (saponification degree: 78-82 mol\%) solution of Sn-PPDE. Gelation takes place by mixing the two solutions. The gel was spread over the glass plate and dried by heating at $60^{\circ} \mathrm{C}$. This dry gel was employed for TADF measurement. No drastic increase of the TADF intensity of this plate by irradiation, just as observed on PVA film, was found. This result could be interpreted as the follows. BoronPVA compound was taken as a didiol type ${ }^{4}$ : tetraoxygens of hydroxyl groups were attached to a boron atom; therefore, the number of free hydroxyl groups extremely decreased. (2) The effect of the crystalline increase of PVA film on TADF intensity was 
<smiles>CCC1CC(CC)O[B-]2(O1)OC(CC)CC(CC)O2</smiles>

Didiol type

examined. The degree of crystallinity of PVA film is well known to be increased by heat treatment ${ }^{4}$, then the PVA film (saponification degree: $78-82 \mathrm{~mol} \%$ ) was heated at $180^{\circ} \mathrm{C}$ for $90 \mathrm{~min}$. After the treatment, the film was allowed to stand for $4 \mathrm{~h}$ in a desiccator. This sample shows gradual increase of TADF intensity for $13 \mathrm{~min}$ by irradiation at $43.5 \pm 0.5^{\circ} \mathrm{C}$. After a continuous increase, TADF intensity reaches maximum. But this increase ratio was vanishingly small (2.8-fold) compared to the untreated film (more than 50-fold). (3) A residual acetyl group lowers the crystallinity of PVA film. Then acetic acid was added to aqueous PVA of Sn-PPDE, because acetic acid was expected to form hydrogen bonds with hydroxyl groups of PVA molecule. These bonds would result in the lowering the crystallinity of PVA. Acetic acid $(99 \%)$ of $0.2 \mathrm{ml}$ or $0.04 \mathrm{ml}$ was added to $100 \mathrm{ml}$ aqueous PVA solutions of Sn-PPDE. The other processes of preparation of PVA film followed the procedure already described. As shown in Fig. 3, the addition of acetic acid has a significant effect on TADF of Sn-PPDE. TADF intensity of Sn-PPDE in PVA film which contains $0.2 \mathrm{ml}$ acetic acid was more than 7-fold the one of Sn-PPDE in non-added PVA film. The addition of acetic acid in a film also brought about a considerable reduction in the time needed to make TADF intensity reach the maximum. To make sure of the effect of protons on TADF intensity, hydrochloric acid was added to PVA film of Sn-PPDE in various concentrations, but no significant effect was observed.

Effect of temperature on TADF of Sn-PPDE in PVA film

The increase rate and ratio of TADF intensity by successive irradiation was also found to be influenced by temperature. As shown in Fig. 4, increasing the temperature drastically shortens the time for TADF intensification to maximum level, and the remarkable increase of TADF intensity with an increase in temperature is also observed. But this increase of TADF intensity as the temperature rises does not obey Arrenius' theory. These results indicate that thermal energy promotes the formation of hydrogen bonds between $\pi$-electron systems of Sn-PPDE and hydroxyl groups of PVA, whose bonds inhibit collisional quenching of excited molecules. During the preparation processes of PVA film of Sn-PPDE, there is a process of dissolving the PVA grains (which have

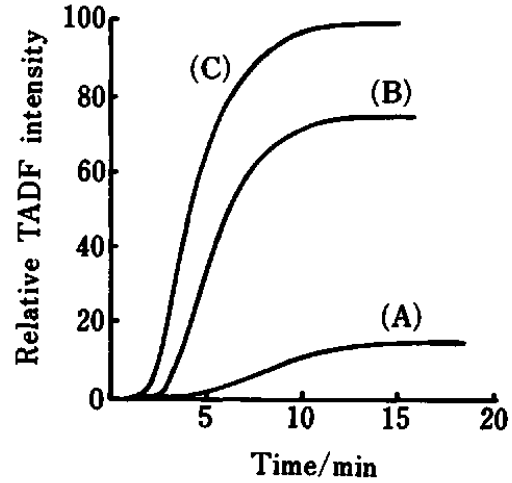

Fig. 3 Dependences of TADF intensity on addition of acetic acid to PVA film. In the preparation process, the following volume of acetic acid $(99 \%)$ was added to $100 \mathrm{ml}$, aqueous PVA solution: (A) $0 \mathrm{ml}$; (B) $0.04 \mathrm{ml}$; (C) $0.2 \mathrm{ml}$.

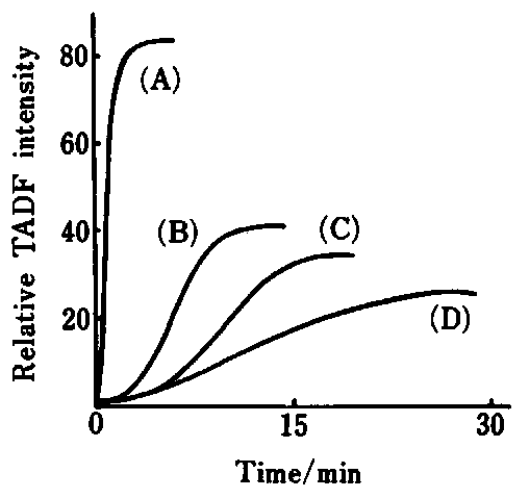

Fig. 4 Effect of temperature on TADF intensity of $\mathrm{Sn}$ PPDE film. TADF was measured at $62.5 \pm 0.5^{\circ} \mathrm{C}(\mathrm{A})$, $42.5 \pm 0.5^{\circ} \mathrm{C}(\mathrm{B}, \mathrm{C})$ or $32 \pm 0.5^{\circ} \mathrm{C}(\mathrm{D})$. Slit widths of excitation and emission of all measurements were $10 \mathrm{~nm}$ and 15 $\mathrm{nm}$, respectively, except that the excitation slit width of (C) was $10 \mathrm{~nm}$.

already been adsorbed by Sn-PPDE) in water. In this process, Sn-PPDE molecule is expected to adsorb to the hydrophobic site of PVA molecule because the molecules of Sn-PPDE do not dissolve in water. The porphyrin rings of Sn-PPDE are located fairly close to the hydroxyl groups of PVA, but few interactions between $\pi$-electron and hydroxyl groups are expected. After the solidification of PVA film by drying, irradiation with blue light appears to promote the formation of hydrogen bonds between the protons of hydroxyl groups and adjacent electron system of SnPPDE, because $\pi^{*}$-orbital is expected to be more polarizable than $\pi$-orbital. Then the effect of temperature on the formation of hydrogen bonds was examined along with the irradiation effect. Flexibility of PVA molecules increases with increasing temperature, so that this increase of flexibility makes it easy to orientate the molecules of Sn-PPDE for the formation of hydrogen bonds with hydroxyl groups of PVA. 
A remarkable TADF increase of Sn-PPDE in films of PA and PAA by successive irradiation with blue light is normally expected because both molecules have a proton which has a capability of forming hydrogen bonds, but in fact remarkable TADF increases were not observed. This might be interpreted as showing that carboxyl groups of PA and amide groups of PAA are too large to form hydrogen bonds, which allows Sn-PPDE to orient suitably in substrate matrix.

The results of this work allow us to have a new understanding of the importance of the matrix for TADF measurement. Functional groups of solid support molecules, which can act as a proton acceptor or donor, hold the key to success for TADF measurements. Especially, the formation of hydrogen bonds between hydroxy groups of PVA molecules and $\pi$-electron systems of Sn-PPDE was found to markedly promote TADF intensity.

\section{References}

1. E. M. Schulman and C. Walling, J. Phys. Chem., 77, 902 (1973).

2. Y. Onoue, K. Hiraki and Y. Nishikawa, Bull. Chem. Soc. Jpn., 54, 2633 (1981).

3. Y. Onoue, K. Hiraki and Y. Nishikawa, Bunseki Kagaku, 31, 169 (1982).

4. K. Nagano, S. Yamane and K. Toyoshima, "Polyvinylalcohol", p. 142, Kōbunshi Kankōkai Co. (1982).

(Received July 21, 1987) (Accepted October 23, 1987) 